\title{
ON PSEUDO-ANOSOV MAPS WHICH EXTEND OVER TWO HANDLEBODIES
}

\author{
by D. D. LONG* \\ (Received 18th October 1988)
}

We show that there is a pair of handlebodies $H_{1}$ and $H_{2}$ with common boundary $F$ with the properties:

(a) There is no essential simple closed curve in $F$ bounding a disc in both $H_{1}$ and $H_{2}$.

(b) Given any positive number, there are essential simple curves $C_{i} i=1,2$ on $F$, bounding discs in $H_{i}$ whose distance apart in the Hausdorff topology on $F$ is less than this positive number.

Such an example has consequences for Heegaard splittings and recognising the 3 -sphere.

1980 Mathematics subject classification (1985 Revision): 57.

\section{Introduction}

Let $\theta: F \rightarrow F$ be an orientation preserving homeomorphism of a closed orientable surface of genus at least two. We say that the map $\theta$ extends over the 3-manifold $M$ if there is an identification $\phi: F \rightarrow \partial M$, and a homeomorphism $\Theta: M \rightarrow M$ so that $\theta=$ $\phi^{-1} \Theta \mid \partial M \phi$. A case of particular interest is when $M$ is a cube-with-handles or "handlebody". A question, attributed to Andrew Casson, which has been around for some time is the following:

Suppose that $\theta$ is a pseudo-Anosov map which extends over handlebodies $M_{1}$ and $M_{2}$, that is to say, there are handlebodies $M_{1}$ and $M_{2}$, identifications $\phi_{i}: F \rightarrow \partial M_{i}$ and homeomorphisms

$$
\Theta_{i}: M_{i} \rightarrow M_{i} \text { so that } \theta=\phi_{i}^{-1} \Theta_{i} \mid \partial M_{i} \phi_{i} \quad i=1,2
$$

Must there be an essential simple closed curve $C$ on $F$ which bounds a disc in both $M_{1}$ and $M_{2}$ ?

We shall indicate some reasons why this is of interest below. The purpose of this note is to show that the answer to this question is negative. It is of course necessary to restrict the curve $C$ to be simple; since nontrivial normal subgroups in a surface group always intersect, so that there is always a (possibly non-simple) loop which is null homotopic in both handlebodies.

One reason for searching for such an example was that it obstructed a possible approach to deciding the question of when a Heegaard splitting is reducible; this was

*Partially supported by NSF Grant No. DMS-870 1422. 
the hope that if for every $\varepsilon$, there were embedded discs $D_{1}$ in $M_{1}$ and $D_{2}$ in $M_{2}$ whose boundaries were $\varepsilon$ close, then there would be a simple curve $C$ on $F$ bounding a disc in both $M_{1}$ and $M_{2}$. More precisely, we have the following. Fix a hyperbolic metric on $F$, and use this to define the Hausdorff metric, $\chi(.,$.$) , on the closed subsets of F$. (See [2].) Combining some knowledge of the action of pseudo-Anosov maps with a result of [9], we deduce:

Corollary 1.4. It is possible to find a pair of handlebodies $M_{1}$ and $M_{2}$ with $\partial M_{1}=\partial M_{2}$ which have the following properties.

(a) There is no simple closed curve bounding a disc in $M_{1}$ and $M_{2}$.

(b) Given any $\varepsilon>0$, there is a pair of essential simple closed curves $C_{1}$ and $C_{2}$ in $F$, with $C_{i}$ bounding a disc in $M_{i}$ and $\chi\left(C_{1}, C_{2}\right)<\varepsilon$.

One of the reasons that such an example is hard to find is that it is by no means easy to give examples of pseudo-Anosov maps which extend a handlebody in essentially different ways. There are two trivial constructions: one involving periodic maps which commute with the pseudo-Anosov, and the other by using pairs of curves which fill the surface and bound discs in more than one handlebody. The second of these is doomed to failure, and the first always seems to fail. Moreover, in general the checking of any potential counterexample is impeded by the fact that there appears to be no algorithm known to determine if a pair of handlebodies share a simple closed curve bounding a disc in each.

We say that $\xi$ exchanges two handlebodies, if there are handlebodies $M_{1}$ and $M_{2}$ so that $\xi$ does not extend over either handlebody, but $\xi^{2}$ extends over both. Then we can also answer the following questions, raised in [10]. Suppose that $\xi$ is a pseudo-Anosov which exchanges a pair of handlebodies. Is there a periodic map commuting with $\xi$ which exchanges them? Part of our construction produces a pseudo-Anosov $\xi$ which exchanges $M_{1}$ and $M_{2}$. However, a calculation shows: $M_{2}$.

Corollary 1.5. There is no periodic map commuting with $\xi$ which exchanges $M_{1}$ and

Thus the answer to this question is also negative. Our example has genus $(F)=4$, and arises from a somewhat complicated extension which arises in nature as a result of a certain knot being ribbon. The proof that our example works relies heavily on two pieces of luck.

\section{The example}

In this section we describe our example; some of the details involved in the computation are deferred until Section 2. The construction involves the monodromy of the (3.5) Turk's head knot, the knot 10* of Conway's tables; displayed as the boundary of its Seifert surface in Fig. 1. This knot is hyperbolic, so that the monodromy $\theta^{\prime}: F^{\prime} \rightarrow F^{\prime}$ 

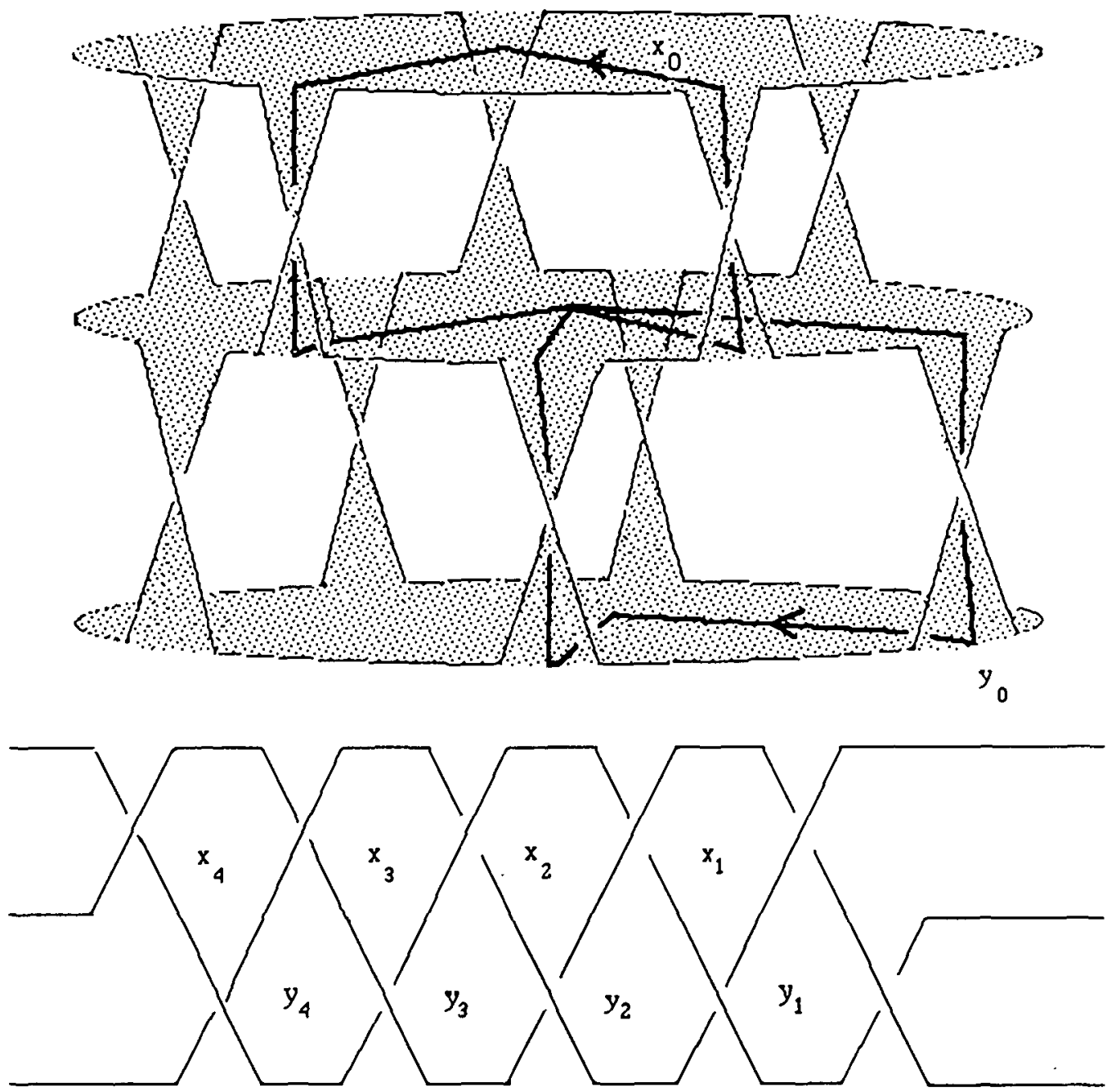

FIGURE 1

of the Seifert surface is pseudo-Anosov. Since we are interested in a map of a closed surface to itself, we cap off $F^{\prime}$ and extend $\theta^{\prime}$ across this disc, to obtain an automorphism $\theta: F \rightarrow F$. We shall prove in Section 2 that this extended map continues to be pseudoAnosov. Observe that the genus of $F$ is four.

We need to describe our generators for the fundamental group of $\pi_{1}\left(F^{\prime}\right)$; this is also done in Fig. 1. The basepoint is considered to be on the central disc and the loops are all of the type shown, with the convention shown in the bottom figure for their numbering.

In the notation of Fig. 1, it is convenient to write: 


$$
\pi_{1}(F)=\left\langle x_{0}, x_{1}, \ldots, x_{4}, y_{0}, y_{1}, \ldots, y_{4}\right| x_{0} \cdot x_{1}, \ldots \cdot x_{4}=y_{0} \cdot y_{1}, \ldots \cdot y_{4}=I, R>
$$

Here $R$ is the relation which comes from capping off the knot; one verifies from Fig. 1 that this is the relation:

$$
1=y_{0} x_{1} y_{3} x_{4} y_{1} x_{2} y_{4} x_{0} y_{2} x_{3}
$$

Lemma 1.1. Use the symbol $\langle\cdot\rangle_{n}$ to denote normal closure in $\pi_{1}(F)$.

Set

$$
\begin{gathered}
N_{1}=\left\langle y_{2} x_{2} x_{0}, y_{3} x_{4} x_{2}^{-1}, y_{1} x_{0}^{-1} x_{4}^{-1} x_{2}^{-1} x_{1} x_{2}, y_{4} x_{2}^{-2} x_{1}^{-1} x_{2}\right\rangle_{n} \\
N_{2}=\left\langle y_{2} x_{2} x_{3} x_{0}^{-1} x_{3}^{-1}, y_{3} x_{3} x_{0} x_{1}, y_{1} x_{2} x_{3}, y_{4} x_{0} x_{4} x_{3}^{-1}\right\rangle_{n}
\end{gathered}
$$

Then there are handlebodies $M_{1}$ and $M_{2}$, whose boundary is $F$ and for which

$$
N_{i}=\operatorname{ker}\left\{i_{*}: \pi_{1}(F) \rightarrow \pi_{1}\left(M_{i}\right)\right\} i=1,2 .
$$

Proof. One checks that in both cases the four words given describe disjoint simple closed curves on the surface $F$. Then the handlebodies are formed by by sewing in 2handles along thin neighbourhoods of these curves and capping off the resulting 2sphere boundary component with a 3-handle.

Lemma 1.2. The map $\theta$ extends over both $M_{1}$ and $M_{2}$.

Proof. It is shown in [3], that this is equivalent to the map $\theta *$ preserving the subgroups $N_{1}$ and $N_{2}$. (The subgroups are normal, so that the choice of basepoint is irrelevant here.) Using the calculations of Section 2, this can be verified.

Remark. We shall indicate where this pair of handlebodies came from and why they satisfy 1.1 and 1.2 in Section 2.

Our example is completed by the following:

Theorem 1.3. There is no simple closed curve representing an element of the subgroup $N_{1} \cap N_{2}$.

Proof. Form a 3-manifold $X$ by glueing together $M_{1}$ and $M_{2}$ along $F$. By the Seifert-Van Kampen theorem, we obtain a presentation of $\pi_{1}(X)=G$, which after eliminating the $y_{i}^{\prime}$ 's has the shape:

Generators. $x_{0}, x_{1}, \ldots, x_{4}$ 
Relations.

$$
\begin{gathered}
x_{0} \cdot x_{1} \ldots \cdot x_{4}=1 \\
R \\
x_{2} x_{0}=x_{2} x_{3} x_{0}^{-1} x_{3}^{-1} \\
x_{4} x_{2}^{-1}=x_{3} x_{0} x_{1} \\
x_{0}^{-1} x_{4}^{-1} x_{2}^{-1} x_{1} x_{2}=x_{2} x_{3} \\
x_{2}^{-2} x_{1}^{-1} x_{2}=x_{0} x_{4} x_{3}^{-1}
\end{gathered}
$$

It follows directly from (1) that we have an equivalent relation:

$$
x_{0}=x_{3} x_{0}^{-1} x_{3}^{-1}
$$

From (2), (1) we deduce:

$$
x_{4} x_{0}=x_{3} x_{0} x_{1} x_{2} x_{3} x_{0}^{-1} x_{3}^{-1}=x_{3}\left(x_{0} x_{1} x_{2} x_{3}\right) x_{0}^{-1} x_{3}^{-1}=x_{3} x_{4}^{-1} x_{0}^{-1} x_{3}^{-1} .
$$

Now using (1)', we deduce that (2) is equivalent to:

$$
x_{4}=x_{3} x_{4}^{-1} x_{3}^{-1} \text {. }
$$

Now from (4), we may write $x_{1}=x_{2} x_{3} x_{4}^{-1} x_{0}^{-1} x_{2}^{-2}$ and use this to substitute into (3), yielding:

$$
x_{0}^{-1} x_{4}^{-1} x_{2}^{-1} x_{2} x_{3} x_{4}^{-1} x_{0}^{-1} x_{2}^{-2} x_{2}=x_{2} x_{3}
$$

Using (1)' and $(2)^{\prime}$ and $(3)^{\prime}$ we deduce from this relation, the new relation:

$$
x_{2}=x_{3} x_{2}^{-1} x_{3}^{-1}
$$

Finally, we remove $x_{1}$ from the relations (1),., (4) altogether by using $x_{0} \cdot x_{1} \ldots x_{4}=1$ in (4), which becomes:

$$
x_{3}^{2} x_{2} x_{4} x_{0} x_{2} x_{4} x_{0}=1
$$

This gives an equivalent presentation for $G$ :

Generators. $\quad x_{0}, x_{2}, x_{4}, x_{3}$ 


\section{Relations.}

$$
\begin{gathered}
R \\
x_{0}=x_{3} x_{0}^{-1} x_{3}^{-1} \\
x_{2}=x_{3} x_{2}^{-1} x_{3}^{-1} \\
x_{4}=x_{3} x_{4}^{-1} x_{3}^{-1} \\
x_{3}^{2} x_{2} x_{4} x_{0} x_{2} x_{4} x_{0}=1 .
\end{gathered}
$$

We now may rewrite the knot relation, by substituting for the $y_{i}$ 's and $x_{1}$. A routine calculation reveals that $R$ is equivalent to:

$$
x_{4} x_{0} x_{2} x_{4} x_{0} x_{2} x_{3}^{2}=1
$$

Observe that $x_{3}^{2}$ is central in $G$; so that the knot relation is equivalent to the last relation in the above list. This was bound to happen; the fundamental group of $X$ does not notice in this construction whether we use $F$ or $F^{\prime}$ since they differ only in 3-handles.

We may now prove Theorem 1.3. First observe that $H_{1}(X)=\mathbf{Z}_{2} \oplus \mathbf{Z}_{2} \oplus \mathbf{Z}_{2} \oplus \mathbf{Z}_{2}$. It follows that the minimal number of generators of $G$ is four. Further, any embedded $S^{2}$ in $X$ is separating, since $H_{1}(X)$ is finite. Recalling that the genus of $F$ is four, we see that if there were a simple closed curve bounding a disc in $M_{1}$ and $M_{2}$ the induced reduction of $X=X_{1} \# X_{2}$ could not have either piece simply connected, else the induced Heegaard splitting on the non simply-connected piece would exhibit $G$ as $a \leqq 3$ generator group.

It follows that if such a curve existed, we could write $G$ as a nontrivial free product $G_{1} * G_{2}$. However, as we have already observed, the element $x_{3}^{2}$ is central in $G$; this cannot happen in a nontrivial free product unless $x_{3}^{2}=1$.

To rule out this possibility, observe that the group $G$ surjects onto the quaternion group $Q_{8}$ which has presentation:

$$
\left\langle-1, i, j, k \mid i^{2}=j^{2}=k^{2}=-1, i j=-j i, i k=-k i, j k=-k j\right\rangle
$$

where we map

$$
x_{3} \rightarrow i x_{0} \rightarrow 1 x_{2} \rightarrow k x_{4} \rightarrow j
$$

In this group $x_{3}$ has order 4 , completing the proof.

We can identify the manifold $X$ with little difficulty. The presence of centre shows that it is likely to be a Seifert fibred space. We can exhibit the fundamental group in the form given in Hempel's book [7] as follows:

Set $x_{2} x_{3}=c_{2}, x_{4} x_{3}=c_{4}, x_{0}^{-1} x_{3}=c_{0}, x_{3}=c_{3}, c=c_{4} c_{0} c_{2} c_{3}$ and $t=x_{3}^{2}$.

Then $G$ has presentation with generators $c_{0}, c_{2}, c_{4}, c_{3}, c, t$ with relations: 
(a) $t$ is central.

(b) $c_{i}^{2}=t$ and $c^{2}=t^{-1}$

(c) $c_{4} c_{0} c_{2} c_{3}=c$.

Thus $X$ is Seifert fibred over a two sphere with 5 exceptional fibres-as an orbifold these are cone points with angle $\pi$, so that the orbifold Euler characteristic is $-1 / 2$.

Proof of 1.4. The result of [4] implies that there is an essential curve bounding a disc in $M_{1}$ which is the union of an arc $\alpha_{+} \cup \alpha_{-}$where $\alpha_{ \pm}$are arcs in the invariant laminations for $\theta$. Similarly, such a curve exists for $M_{2}$. Moreover, one can check that these arcs can be chosen so that they do not cut across the principal regions for the laminations, that is, they are not subarcs of isolated leaves. (The existence of such curves can also be deduced from [9].) Denoting these two curves by $A_{1}$ and $A_{2}$ we now have from [2], Theorem 4.1, that $\theta^{k} A_{i} \rightarrow L^{+}$for $i=1,2$, where $L^{+}$is the unique perfect stable lamination left invariant by $\theta$, and the convergence is in the Hausdorff topology. The corollary now follows.

Proof of 1.5. Suppose that such a map existed; call it $\omega$ say. Since $\omega$ commutes with $\theta$, it must fix the invariant foliations of $\theta$; moreover, it must also preserve the measure on these foliations. The latter is because if $\mu$ is the invariant measure on the stable foliation say, we can form an $\omega$-invariant measure by $M=\mu+\omega^{*} \mu+\cdots+\left(\omega^{*}\right)^{n-1} \mu$. Since this is an invariant measure on the stable foliation, unique ergodicity gives $M=k \mu$. Thus $\omega$ is an isometry of the affine structure defined by the union of the stable and unstable measure foliations. In particular, the curve $A_{1}$ constructed above must be carried to a curve which bounds a disc in $M_{2}$ and is the union of two straight lines of the same length. One can check explicitly that no such curve exists.

\section{Some computations}

In this section we show how one can perform the computations alluded to in the previous sections.

The knot $K$ which we consider is the (3.5) Turk's Head knot of Fig. 1. It is alternatively described as the braid closure of $\left(\sigma_{1} \sigma_{2}^{-1}\right)^{5}$. This is a homogeneous braid in the sense of [11], so that $K$ is fibred, with fibre surface $F^{\prime}$, visible in Fig. 1. The knot of Fig. 1 clearly has a 5 -fold symmetry coming from rotation about a vertical axis; this symmetry induces a map $\omega: F^{\prime} \rightarrow F^{\prime}$ which has three fixed points and carries the boundary circle to itself, so that $\omega$ extends as a map of the closed surface $F$ to itself. Clearly $\omega$ and the monodromy of $K$ commute.

We now prove that the monodromy is pseudo-Anosov in the following way. There is a branched covering $p: F \rightarrow F / \omega$; where there are four branch points, each of order 5 . The surface $F / \omega$ is easily seen to be topologically a 2 -sphere and can be considered metrically as an orbifold with 4 cone points, each of angle $\pi$. This orbifold is flat; in fact denoting the flat torus by $T$, this has a central involution $\tau$, and we have a metric covering $T \rightarrow T / \tau=F / \omega$. This gives a correspondence between maps of the torus to itself and orbifold maps of $F / \omega$. 
The methods of Goldsmith [6] apply to this situation (indeed, she works out this example, though not in quite this notation) and we deduce that the monodromy $\theta: F \rightarrow F$ comes from lifting the linear map:

$$
\theta=\left[\begin{array}{ll}
2 & 1 \\
1 & 1
\end{array}\right]: F / \omega \rightarrow F / \omega
$$

Since this map is a hyperbolic map of the torus, the lifted map $\theta$ is pseudo-Anosov; it has the same local properties. (See [5].) This description enables one to visualise the monodromy on $F$ in a very concrete way, but we shall not need to do this, we refer to [8].

More importantly, the knot $K$ is ribbon, so that by a theorem of Casson-Gordon [3], the map $\theta$ extends over a handlebody; and by examining the ribbon move, one sees that the element $x_{2} y_{0}$ bounds a disc in this handlebody. Below we shall give a description of the action of $\theta_{*}$ on $\pi_{1}(F)$; knowledge of one nonseparating curve defines the whole handlebody, since the characteristic polynomial of $\theta_{*}: H_{1}(F) \rightarrow H_{1}(F)$ factorises as $f(t) f\left(t^{-1}\right)$, with $f(t)$ irreducible over $\mathbf{Z}$. This gives handlebody $M_{1}$ of Lemma 1.1 .

One of the surprising features given the highly symmetric nature of $K$ is that the handlebody $M_{1}$ is so asymmetric. We digress to observe that this is actually forced to be the case, but since we make no use of this fact, we only sketch the proof:

Proposition 2.1. There is no compression body over which both $\theta$ and $\omega$ extend.

Proof. One computes easily that $\omega_{*}$ has characteristic polynomial $g(t)^{2}$, where $g(t)=t^{4}+r^{3}+\cdots+1$; which is $\mathbf{Z}$ irreducible. If follows that if $\omega$ compresses at all, it must either be a handlebody or involve just one 2-handle. The latter is impossible if the pseudo-Anosov is also to extend over the compression, since the core of the 2-handle would have to be preserved up to free homotopy. But now an examination of the fixed point data [1] shows that $\omega$ cannot extend over any 3-manifold.

Notice that this already gives five handlebodies over which $\theta$ extends, since $\omega^{r} N_{1}$ must be different from $N_{1}$ for $0<r<5$. However the reader may check that every such pair gives a reducible Heegaard splitting.

The second handlebody of Lemma 1.1 arises in the following way. Notice that we have an orientation reversing square root in $G L(2, \mathbf{Z})$ for the matrix $\theta$. Writing $\xi^{2}=\theta$, it is easy to check that $\xi$ also lifts to $F$, and that choosing an appropriate lift, also denoted $\xi$, we may arrange $\xi^{2}=\theta$ as maps of $F$. Thus $\theta$ also extends over the handlebody $\xi N_{1}$. This gives another five handlebodies. In the notation of Lemma 1.1, $N_{2}=\omega^{2} \xi N_{1}$. Empirically one finds that this choice is the only pair which yields an irreducible Heegaard splitting.

It remains only to show how to obtain the action of $\theta_{*}$ on $\pi_{1}(F)$. It turns out that a trick makes this fairly easy. We refer to Fig. 2, and regard the basepoint and surface as living behind the page; so that we are using the Wirtinger under-presentation. Write $a^{b}$ for $b^{-1} a b$. 


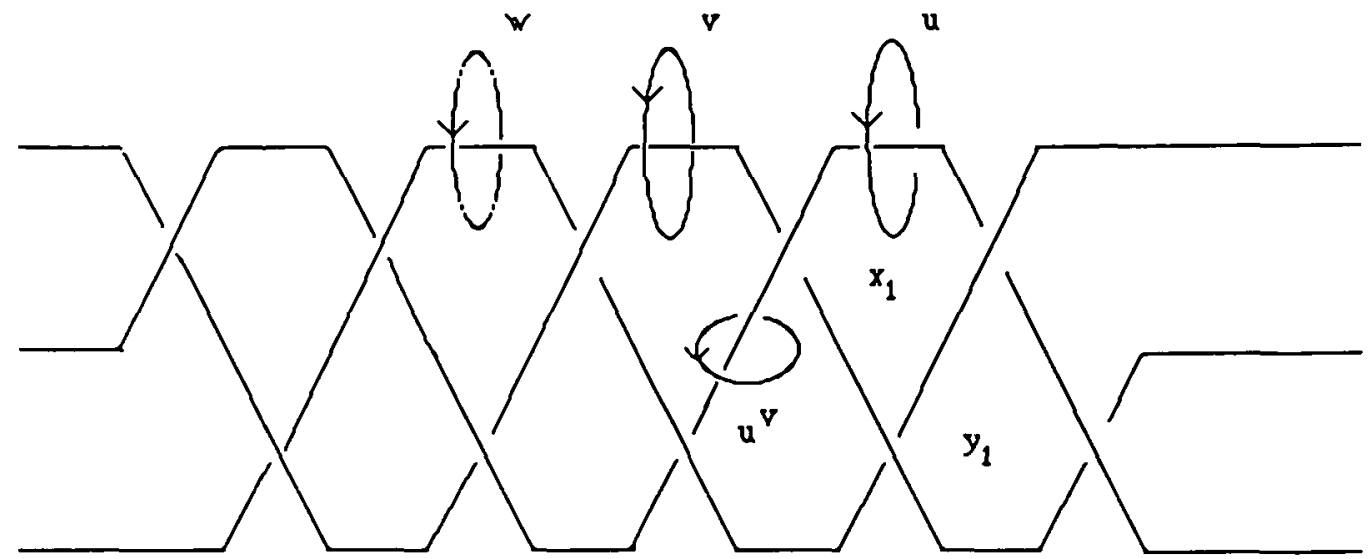

FIGURE 2

Then the action of the monodromy is given by conjugation by a meridian; initially we use $v$. We see that:

$$
\begin{gathered}
x_{1}=u^{-1} v \quad y_{2}=\left(u^{-1}\right)^{v} \cdot w=v^{-1} u^{-1} v w \\
x_{2}=v^{-1} w \quad y_{3}=u^{v}\left(v^{-1}\right)^{w}=v^{-1} u v w^{-1} v^{-1} w \\
\theta x_{2}=v x_{2} v^{-1}=w v^{-1}=x_{2} y_{3}^{-1} x_{1}^{-1} \\
\theta y_{2}=v y_{2} v^{-1}=u^{-1} v w v^{-1}=x_{1} x_{2} y_{3}^{-1} x_{1}^{-1}
\end{gathered}
$$

Now we may compute the action of the monodromy on the remainder of the generators by chicanery. Notice that we could have used $w$ to compute the monodromy, and therefore by symmetry we have:

$$
\theta_{w}\left(x_{3}\right)=x_{3} y_{4}^{-1} x_{2}^{-1}
$$

But

$$
\theta_{w}\left(x_{3}\right)=w x_{3} w^{-1}=\left(w v^{-1}\right) v x_{3} v^{-1}\left(v w^{-1}\right)=x_{2} y_{3}^{-1} x_{1}^{-1} \theta x_{3}\left(x_{2} y_{3}^{-1} x_{1}^{-1}\right)^{-1}
$$

Hence

$$
\theta x_{3}=x_{1} y_{3} x_{2}^{-1} x_{3} y_{4}^{-1} x_{2}^{-1} \cdot x_{2} y_{3}^{-1} x_{1}^{-1}=x_{1} y_{3} x_{2}^{-1} x_{3} y_{4}^{-1} y_{3}^{-1} x_{1}^{-1}
$$

This gives an inductive method of computing the monodromy which actually works for any $(3, k)$ Turk's Head knot. One finds: 


$$
\begin{aligned}
& \theta x_{0}=x_{1} y_{2}^{-1} y_{1}^{-1} x_{4}^{-1} x_{0} y_{2} x_{1}^{-1} \quad \theta y_{0}=x_{1} y_{2}^{-1} y_{1}^{-1} x_{0} y_{2} x_{1}^{-1} \\
& \theta x_{1}=x_{1} y_{2}^{-1} x_{0}^{-1} \quad \theta y_{1}=x_{1} y_{2}^{-1} \\
& \theta x_{2}=x_{2} y_{3}^{-1} x_{1}^{-1} \quad \theta y_{2}=x_{1} x_{2} y_{3}^{-1} x_{1}^{-1} \\
& \theta x_{3}=x_{1} y_{3} x_{2}^{-1} x_{3} y_{4}^{-1} y_{3}^{-1} x_{1}^{-1} \quad \theta y_{3}=x_{1} y_{3} x_{3} y_{4}^{-1} y_{3}^{-1} x_{1}^{-1} \\
& \theta x_{4}=x_{1} y_{3} y_{4} x_{3}^{-1} x_{4} y_{1} y_{2} x_{1}^{-1} \quad \theta y_{4}=x_{1} y_{3} y_{4} x_{4} y_{1} y_{2} x_{1}^{-1} \text {. }
\end{aligned}
$$

\section{REFERENCES}

1. F. Bonahon, Cobordism of automorphisms of surfaces, Ann. Sci. École, Norm. Sup. (4), 16 (1983), 237-270.

2. A. J. CAsson, Automorphisms after Nielsen and Thurston (LMS Lecture Notes, 15, Cambridge, 1988).

3. A. J. CAsson and C. McA. Gordon, A loop theorem for duality spaces and fibred ribbon knots, Invent. Math. 74 (1983), 119-139.

4. A. J. CAsson and D. D. Long, Algorithmic compression of surface automorphisms, Invent. Math. 81 (1985), 295-303.

5. A. Fathi et al., Travaux de Thurston sur les surfaces, Astérisque 66-67.

6. D. Goldsmith, Symmetric fibered links, Knots, groups and 3-manifolds (Ann. of Math. Studies 84, Princeton, 1975).

7. J. Hempel, 3-Manifolds (Ann. of Math. Studies 86, Princeton University Press, 1976).

8. D. D. Long, Cobordism of knot and surface automorphisms (Thesis, Cambridge University, 1983).

9. D. D. LonG, Discs in compression bodies, Pacific J. Math. 122 (1986), 129-146.

10. D. D. Long, Bounding laminations, Duke J. Math. 56 (1988), 1-16.

11. J. Stallings, Constructions of fibred knots and links (Proc. Symp. in Pure Math. 32, Part II, 1978), 55-60.

Department of Mathematics

UNIVERSITY OF CALIFORNIA

Santa Barbara, CA. 93106

USA 